\author{
Elyazid Mouhsine \\ Pierre François Leyvraz \\ Olivier Borens \\ Michel Ribordy \\ Yvan Arlettaz \\ Raffaele Garofalo
}

\section{Acute fractures of medial and lateral great toe sesamoids in an athlete}

Keywords Sesamoids · Great toe · Acute fracture . Trauma
Received: 20 August 2003

Accepted: 11 October 2003

Published online: 10 January 2004

(C) Springer-Verlag 2004

E. Mouhsine ( $\bowtie) \cdot$ P. F. Leyvraz

O. Borens · M. Ribordy · Y. Arlettaz

R. Garofalo

Department of Orthopaedic Surgery

and Traumatology,

Centre Hospitalier Universitaire Vaudois,

1011 Lausanne, Switzerland

Tel.: +41-21-3142791,

Fax: +41-21-3142974,

e-mail: elyazid.mouhsine@hosr.hospvd.ch
Abstract We report a case of acute fracture of both sesamoids of the great toe in an athlete. The fractures healed uneventfully after non-surgical treatment.

\section{Introduction}

The medial and lateral sesamoids are two important components of the first metatarsophalangeal (MTP) joint complex, due to their important role in absorbing weight-bearing pressure, in friction reduction, and in tendon protection [7]. During the normal gait cycle, the center of pressure progresses along the plantar aspect of the foot from the heel at heel strike to the toes at toe off. At toe off in particular the center of pressure is located underneath the first MTP joint [4]. The sesamoids bear up to three times of body weight during a normal cycle, and medial sesamoid bears the majority of this force [5]. These pressures are even higher during high-velocity and high-impact athletic activity. Because the medial sesamoid bears most of the force under the first metatarsal head, it is most likely to be injured $[1,3,4,7,9]$.

To the best of our knowledge this is the first report of an acute fracture occurring simultaneously in both sesamoid bones of the first MTP joint.

\section{Case report}

A 30-year-old male, a $110 \mathrm{~m}$ hurdler, presented at our trauma center with sharp pain located at the plantar aspect of the first ray of the left foot, which had occurred during training. The patient reported the appearance of a sharp pain and the sensation of a crack in his plantar forefoot on landing after a leap. Due to the pain he could not continue the training. On physical examination there was a localized sharp pain on pressure, and weight bearing was possible only using the lateral aspect of the foot. The plantar aspect of the first MTP joint was swollen and tender. Passive dorsiflexion of the MTP joint exacerbated the pain. Radiographs and 3D CT-scan showed a comminuted fracture of both sesamoid bones (Figs. 1 and 2).

Conservative treatment was chosen, consisting of protected weight-bearing in a short leg cast with a walking heel for six weeks and rest. After this time the cast was removed, a subsequent 6-week protection with a metatarsal pad was carried out, and a rehabilitation program consisting of weight bearing and proprioceptive exercises was started. At clinical examination after 12 weeks, weight bearing was pain-free. 


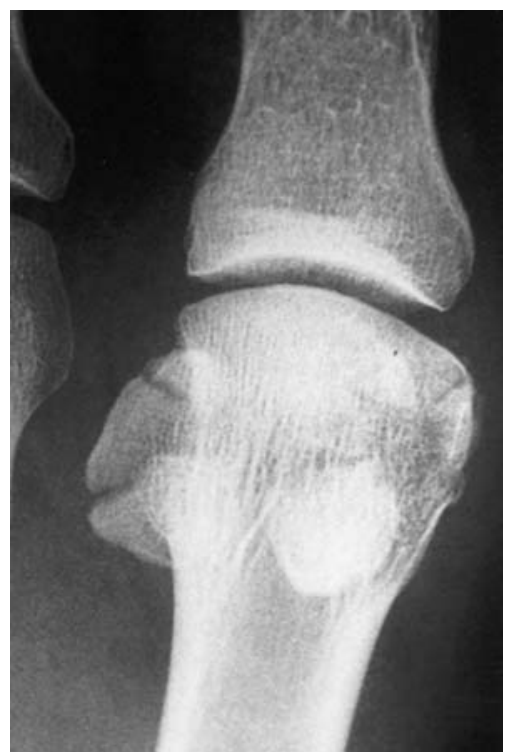

Fig. 1 Anteroposterior radiograph of the first MTP joint with sesamoid fractures

\section{Discussion}

The mechanism leading to sesamoid acute fracture can include a fall from a height, a crush injury to the forefoot, or sudden loading of the first MTP joint [3,5]. Sometimes, forced great-toe dorsiflexion and abduction result in fracture by indirect mechanism [5]. The medial sesamoid tends to fracture more often than the lateral one. The lateral sesamoid is less involved in fractures because only the medial half of the first metatarsus presses on the ground during walking, as proposed by some authors [8]. Fractures are usually transverse but as in our case may be comminuted following direct trauma [2].

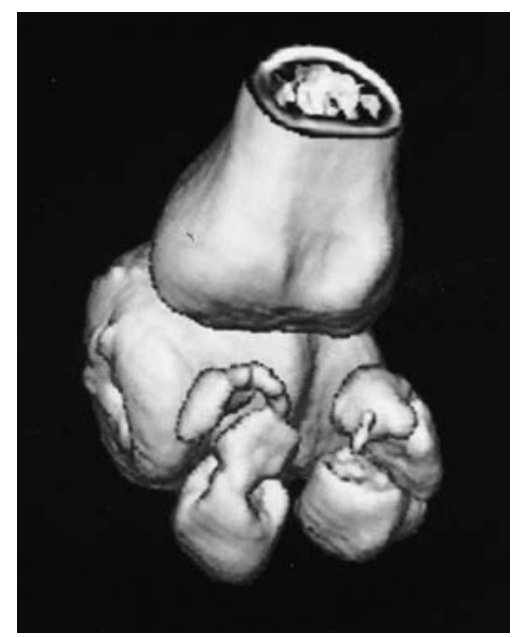

Fig. 2 3D CT-scan of the fracture of both sesamoids

In our case the diagnosis was evident, and a 3D CT-scan was performed to better understand the degree of comminution of the fracture, and for decision-making regarding the treatment.

Different non-surgical care for sesamoid fracture has been proposed, including a short leg cast, a metatarsal bar, a metatarsal pad, and a stiff-soled shoe with pads to protect the sesamoid $[2,5,6]$.

We chose a short leg walking cast with a walking heel placed to decrease weight on the first metatarsal head, as proposed by DeLee [2]. As proposed by McBryde and Anderson [6], a further 6 weeks of protection with a metatarsal pad was carried out. We chose this therapeutic strategy because both sesamoids were involved, and because the fractures were comminuted. The patient resumed his athletic activity after 4 months, and a painless loss of range of motion of first MTP was found.

\section{References}

1. Biedert R, Hintermann B (2003) Stress fractures of the medial great toe sesamoids in athletes. Foot Ankle Int 24:137-141

2. DeLee JC (1993) Fractures and dislocation of the foot. In: Mann RA, Coughlin MJ (eds) Surgery of the foot and ankle. Mosby Year-Book, St Louis, pp 14651703
3. Feldman F, Pochaczevsky R, Hecht H (1970) The case of the wandering sesamoid and other sesamoid afflictions. Radiology 96:275-283

4. Hockenbury RT (1999) Forefoot problems in athletes. Med Sci Sports Exerc 31:448-458

5. Jahss MH (1981) The sesamoids of the hallux. Clin Orthop 157:88-97

6. McBryde AM Jr, Anderson RB (1988) Sesamoid foot problems in the athlete. Clin Sports Med 7:51-60
7. Richardson EG (1987) Injuries to the hallucal sesamoids in the athlete. Foot Ankle 7:229-244

8. Rinaldi F, Magistroni A (1966) Considerazioni sulla frattura dei sesamoidi dell'alluce. Minerva Ortopedica 17:125-128

9. Zinman H, Keret D, Reis ND (1981) Fracture of the medial sesamoid bone of the hallux. J Trauma 21:581-582 